\title{
Ameliorative effect of Raphanus sativus and Cassia angustifolia in Experimentally Induced Hyperlipidemia and Cardiovascular Risk Reduction
}

\author{
Deepti Kaushalkumar Jani ${ }^{\star}$, Sunita Goswami ${ }^{2}$ \\ ${ }^{1}$ Department of Pharmacology, Babaria Institute of Pharmacy, Varnama, Vadodara, \\ Gujarat, India \\ ${ }^{2}$ Department of Pharmacology, L.M. College of Pharmacy, Ahmadabad, Gujarat, India
}

\begin{abstract}
Hyperlipidemia is considered to be one of the greatest risk factors of cardiovascular diseases. Increase in number of hyperlipidemic and cardiac patients globally; emphasize the need of new therapies to reduce disease burden. The objective of current study is to investigate effect of Raphanus sativus and Cassia angustifolia extracts on experimentally induced hyperlipidemia and cardiovascular risk reduction. Phytochemical study of extracts was done as per standard procedures. As per Organisation for Economic Co-operation and Development (OECD) 420 guideline, single dose acute oral toxicity study performed using both extracts. Hyperlipidemia was induced in rats using Poloxamer 407 (P407) and blood samples were collected at 0,24 and $48 \mathrm{~h}$ after P407 administration. Cardiovascular risk was assessed by analysing atherogenic indices. Results indicate that no sign of toxicity was observed with chosen extracts. Sgnificant reduction in total cholesterol (TC), triglyceride (TG), low density lipoprotein (LDL), non HDL-C (non-high density lipoprotein cholesterol) and very low density lipoprotein (VLDL) was observed in extract treated groups of P407 induced hyperlipidemia. Significant decrease in atherogenic indices was observed in extract treated groups. These results were comparable with the results of Atorvastain treated group. On the basis of results, it is concluded that both the extracts of Raphanus sativus and Cassia angustifolia were found to ameliorate hyperlipidemia and risk of cardiovascular disorders.
\end{abstract}

Key words : hyperlipidemia, poloxamer 407, hypertriglyceridemia.

\section{Introduction}

Hyperlipidemia is characterized by elevated levels of blood cholesterol. As per WHO, 2.6 million deaths and 29.7 million disabilities were estimated to cause by elevated cholesterol level ${ }^{1}$. Elevated cholesterol is a major cause of coronary heart disease and fatty liver disease, resulting in increased disease burden in both the developed and developing countries ${ }^{1-3}$.

Herbal medicine is cheaper and easily available option for the treatment of hyperlipidemia ${ }^{4}$. Current scenario of herbal drugs indicates increase in demand and utilization of herbal treatments, so it is worthwhile to screen plant drugs for the treatment of widely spread diseases and disorders. 
In this study two plants are chosen to screen i.e. Raphanus sativus and Cassia angustifolia.

Raphanus sativus Linn. (Brassicaceae) is widely grown herb all over the world and has been used for more than 1,500 years. It contains 4-(methylthio)-3-butenyl isothiocyanate, allyl isothiocayanate, benzyl isothiocyanate, phenethyl isothiocyanate, kaempherol glycosides, peroxidases and antioxidants ${ }^{5}$. Reported activities of Raphanus sativus leaves are antidiabetic ${ }^{6}$, antiulcer ${ }^{7}$, anti-inflammatory ${ }^{8}$, laxative ${ }^{9}$ and antioxidant ${ }^{10}$.

Cassia angustifolia Vahl. (Caesalpiniaceae) grows widely in India and other tropical countries. According to ayurveda, the leaves are laxative, anthelmintic, liver tonic and cardiotonic ${ }^{11}$. Leaves contain tinnevellin glycoside, isorhamnetin-3-O-beta-gentiobioside, apigenin-6,8-di-C-glycoside, emodin-8-O-beta-Dglucopyranoside, kaempferol, aloe emodin, D-3-O-methylinositol and sucrose ${ }^{12}$. Reported activities of Cassia angustifolia leaves are antioxidant ${ }^{13}$, hepatoprotective ${ }^{14}$ and pancreatic lipase inhibitor ${ }^{15}$.

Reported activities of chosen plants as well as increase demand of herbal treatments formed the basis of current study. This study is planned to screen Raphanus sativus and Cassia angustifolia extracts for antihyperlipidemic activity and effect on cardiac risk reduction.

\section{Experimental}

\section{Plant material collection and authentication}

Raphanus sativus Linn. leaves were collected by field collection and authentication was done at K.N.K. College of Horticulture, Mandsaur, M.P., India. Cassia angustifolia Vahl. leaves extract was obtained as a gift sample from Vasu Research Center, Gujarat.

\section{Preparation of extract}

Hydroalcoholic extract of Raphanus sativus leaves and aqueous extract of Cassia angustifolia leaves, was prepared by cold maceration method.

\section{Animals and study approval}

Sprague-dawley rats weighing 200-240 g of either sex were used. The animals were housed under standard environmental conditions (temperature $22 \pm 2^{\circ} \mathrm{c}$; humidity 50-60\%) with $12 \mathrm{~h}$ light/ dark cycle. Animals were provided rat pellet diet (Pranav Agro Industries Ltd., India) and water ad libitum. This study was approved by the institutional animal ethical committee. All study procedures were performed as per the guidelines of the Committee for the Purpose of Control and Supervision of Experiments on Animals (CPCSEA).

\section{Phytochemical analysis}

Phytochemical tests were performed according to standard procedures to identify the chemical constituents of both the extracts ${ }^{16-18}$.

\section{Acute oral toxicity study}

Acute oral toxicity study was performed as per OECD 420 guidelines for both extracts.

\section{P407-induced hyperlipidemia}

Animals were divided into five groups (n=06): Group I: VC (1\% CMC, p.o.) Group II: P407 (1g/kg, i.p.) Group III: RS4 (P407+Raphanus sativus extract, $400 \mathrm{mg} / \mathrm{kg}$, p.o.), Group IV: CA4 (P407+Cassia angustifolia extract, $400 \mathrm{mg} / \mathrm{kg}$, p.o.) and Group V: AT (P407+Atorvastatin, $75 \mathrm{mg} / \mathrm{kg}$, p.o.). Drug treatments were started orally once daily two weeks before $\mathrm{P} 407$ administration to respective groups ${ }^{19,20}$. 


\section{Blood sampling}

Blood samples were collected from fasted animals on $0,24 \& 48 \mathrm{~h}$ after P407 administration for the estimation of lipid parameters. Collected blood was allowed to clot at room temperature for 20-25 minutes and centrifuged for 20 min. at 2000 r.p.m. to obtain clear supernatant serum for estimations.

\section{Study parameters}

Serum concentration of TC, TG and HDL was measured by assay procedure mentioned in biochemical kits using semiautoanalyzer (Microlab). The non-HDL cholesterol, LDL, VLDL and atherogenic indices were calculated using the following formulae ${ }^{21-22}$ :

Non-HDL Cholesterol $=$ TC - HDL,$\quad$ VLDL $=0.166(T G)$

$\mathrm{LDL}=\mathrm{LDL}-\mathrm{C}(\mathrm{mg} / \mathrm{dl})=$ Non-HDL-C $\times 90 \%-\mathrm{TG} \times 10 \%$

\section{Atherogenic indices ${ }^{23}$ :}

Castelli Risk Index-I (CRI-I) = TC/HDL, Castelli Risk Index-II (CRI-II $)=$ LDL/HDL

Atherogenic Coefficient $(\mathrm{AC})=(\mathrm{TC}-\mathrm{HDL}) / \mathrm{HDL}$,

Atherogenic Index of plasma (AIP) $=$ Log (TG/HDL-C)

\section{Data analysis}

Data were expressed as mean \pm standard error of mean (mean \pm SEM). Data were analysed using two way analysis of variance (ANOVA) followed by Bonferroni Multiple Comparison Test. Values of $p<0.05$ were considered as statistically significant.

\section{Results}

\section{Phytochemical analysis}

Phytochemical analysis showed presence of flavonoids, saponins, glycosides and tannins in both extracts.

\section{Acute oral toxicity study}

All the animals used in toxicity study, were observed for 14 days and no sign of toxicity was observed during toxicity study. No structural abnormality was found during necropsy study.

\section{Effect of extracts on serum lipid profile}

In P407-induced hyperlipidemia model, significant increase $(\mathrm{P}<0.001)$ was observed in TG, TC, LDL, VLDL, non-HDL-C and HDL level of P407 group at $24 \mathrm{~h}$ and $48 \mathrm{~h}$ as compared to VC group while significant reduction was observed in treatment groups as compared to P407 group (Fig. Ia, Ib, Ic, Id, Ie, If). No significant change was observed in HDL level in all treatment groups and in TG and VLDL level at $24 \mathrm{~h}$ and in LDL level at $48 \mathrm{~h}$ in CA4 group as compared to P407 group (Fig. Ib, Id, Ie). 

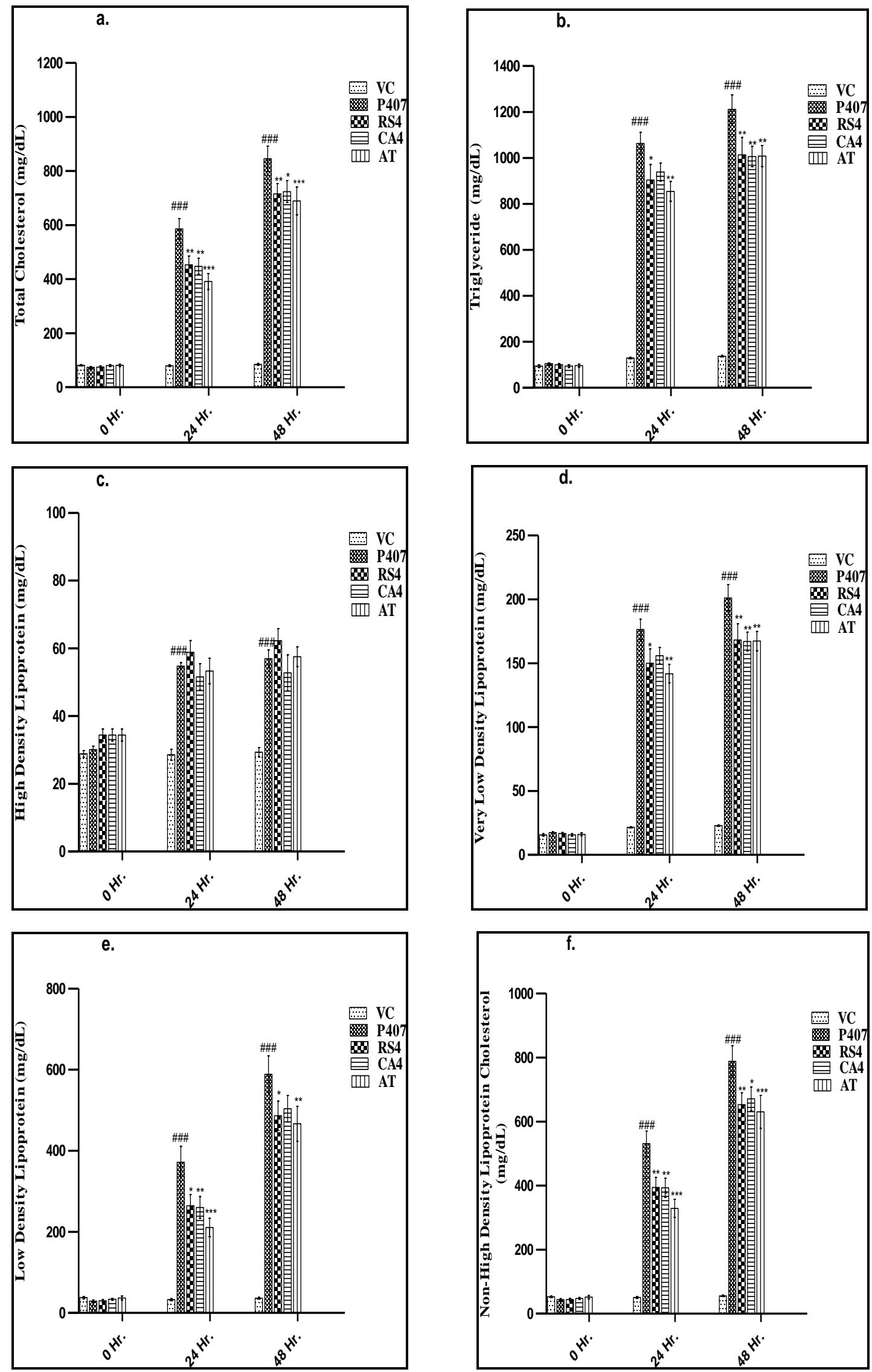

Fig. I: Effect of Raphanus sativus and Cassia angustifolia on lipid profile in Poloxamer-407 induced hyperlipidemia 
Values are expressed as Mean \pm SEM ( $\mathrm{n}=6$ ). Data analyzed by two way ANOVA followed by Bonferroni

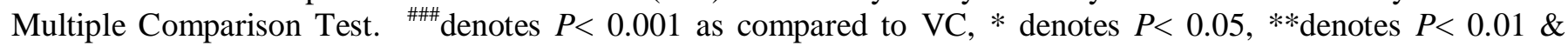
$* * *$ denotes $P<0.001$ as compared to P407. VC-Vehicle control, P407-Poloxamer-407, RS4- Raphanus sativus extract (400 mg/kg), CA4- Cassia angustifolia extract (400 mg/kg), AT-Atorvastatin

\section{Effect of extracts on atherogenic indices}

In P407-induced hyperlipidemia model, significant increase $(\mathrm{P}<0.001)$ was observed in CRI-I, CRI-II, $\mathrm{AC}$ and AIP of P407 group at $24 \mathrm{~h}$ and $48 \mathrm{~h}$ as compared to VC group while significant reduction was observed in treatment groups as compared to P407 group (Fig. IIa, IIb, IIc, IId). No significant change was observed in CRI-I (48 h), CRI-II (48 h), AC (48 h), AIP (24 h, 48 h) in CA4 group and in AIP (24 h) in RS4 group as compared to P407 group (Fig. IIa, IIb, IIc, IId).
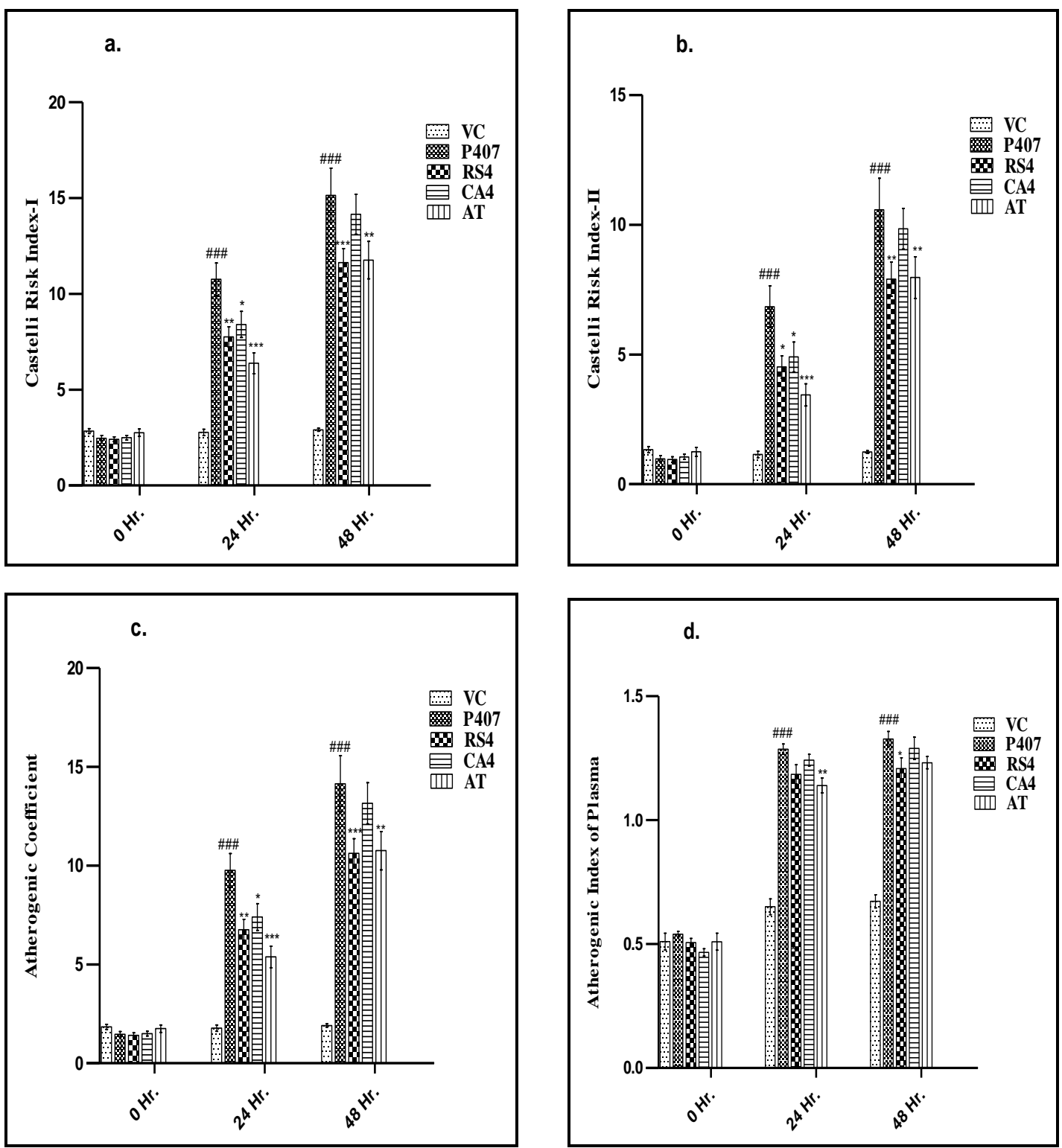

Fig. II: Effect of Raphanus sativus and Cassia angustifolia on atherogenic indices in Poloxamer-407 induced hyperlipidemia

Values are expressed as Mean \pm SEM (n=6). Data analyzed by two way ANOVA followed by Bonferroni Multiple Comparison Test. \#\# denotes $P<0.001$ as compared to VC, * denotes $P<0.05$, **denotes $P<0.01 \&$ ***denotes $P<0.001$ as compared to P407. VC-Vehicle control, P407-Poloxamer-407, RS4- Raphanus sativus extract (400 mg/kg), CA4- Cassia angustifolia extract (400 mg/kg), AT-Atorvastatin 


\section{Discussion}

In the current study, Poloxamer 407 induced hyperlipidemia is selected for screening of herbal drugs, as this is reliable, rapid, convenient and low cost model for screening of antihyperlipidemic activity ${ }^{24}$. Dose of poloxamer 407 used i.e. $1 \mathrm{~g} / \mathrm{kg}$ was found suitable dose for hyperlipidemia induction, since lipid levels were significantly increased at 24 and $48 \mathrm{~h}$ after P407 administration than the control. Our finding in relation to LDL, HDL, TG and TC levels was comparable with the work reported by Chaudhari \& Brocks, $2013^{19}$ previously.

P407 is a non-ionic surfactant, used to produce hyperlipidemia in rats. Hypertriglyceridemia and hypercholesterolemia induced by P407 are due to inhibition of TG degradation by inhibition of lipoprotein lipase enzyme and indirect activation of HMG-CoA reductase respectively ${ }^{24,25}$. This reported mechanism for P407-induced hyperlipidemia points toward the possibility of activation of the lipoprotein lipase or inhibition of HMG-CoA reductase as possible reason for antihyperlipidemic activity of extracts.

Atherogenic indices are powerful indicators of cardiovascular disease. Increase in value of atherogenic indices increases the risk of cardiovascular diseases ${ }^{26}$. Administration of chosen extracts significantly reduced these indices as compared to P407 group, which indicates decrease in risk of cardiovascular diseases in the treatment groups.

Phytochemical constituents possess significant role in the beneficial effects herbal medicines. Both flavonoids and saponins are lipid lowering agents ${ }^{27}$. Flavonoids are antioxidants and may therefore reduce the oxidation of LDL cholesterol. Oxidized LDL is thought to be involved in the development of atherosclerotic diseases $^{28}$. As phytochemical study revealed presence of flavonoids and saponins in the both extracts, their antihyperlipidemic effect and reduction in cardiovascular risk may be due to these phytoconstituents.

\section{Conclusion}

It is concluded that the both the extracts, Raphanus sativus and Cassia angustifolia, significantly improved hyperlipidemia and reduced atherogenic indices, thus reduced risk of cardiovascular diseases.

\section{Acknowledgement}

We express deep gratitude to the Animal Resource department, Sun Pharma Advanced Research Center, Vadodara, India for providing animals for research purpose. We are thankful to Vasu Research Center, Vadodara, India for help in herbal drug procurement.

\section{References}

1. Global Health Observatory (GHO) data, 'WHO | Mean Cholesterol', WHO, 2015. <http://www.who.int/gho/ncd/risk_factors/cholesterol_mean_text/en/>.

2. V. Dhulasavant, S. Shinde, M. Pawar and N.S. Naikwade, Int. J. Pharm. Tech. Res., 2, 2517 (2010).

3. S. Sharma, R.K. Roy and B. Shrivastava, Int. J. Pharm. Tech. Res., 7, 540 (2015).

4. T. Ananthi, I.J. Barvin and M. Chitra, Int. J. Pharm. Tech. Res., 6, 1368 (2014).

5. S.-W. Lee, K.-M. Yang, J.-K. Kim, B.-H. Nam, C.-M. Lee, M.-H. Jeong, S.-Y. Seo, G.-Y. Kim and W.S. Jo, Toxicol. Res., 28, 165 (2012); doi:10.5487/TR.2012.28.3.165.

6. $\quad$ S.P. Macharla, V. Goli and S.R. Sattla, J. Chem. Pharm. Res., 4, 1519 (2012).

7. P.G. Waterman, Biochem. Syst. Ecol., 21, 849 (1993); doi:10.1016/0305-1978(93)90098-C.

8. H.J. Park and M. Song, Prev. Nutr. Food Sci., 22, 50 (2017); doi:10.3746/pnf.2017.22.1.50.

9. P. Dande, A. Vaidya and P. Arora, Asian J. Pharmaceut. Clinical Res., 7, 120 (2014).

10. S.S. Beevi, M.L. Narasu and B.B. Gowda, Plant Foods Hum. Nutr., 65, 8 (2010); doi:10.1007/s11130009-0148-6. 
11. S. Sundaramoorthy, G. Shylaja, A. Sathiavelu and M. Sathiavelu, J. Pharmaceut. Sci. Res., 8, 260 (2016).

12. Q.P. Wu, Z.J. Wang, M.H. Fu, L.Y. Tang, Y. He, J. Fang and Q.F. Gong, Zhong Yao Cai, 30, 1250 (2007).

13. A.Q. Laghari, S. Memon, A. Nelofar and A.H. Laghari, Am. J. Anal. Chem., 2, 871 (2011); doi:10.4236/ajac.2011.28100.

14. R. Shanmugasundaram, K.D. Velusamy, P.S. Tresina, A. Maruthupandian and V.R. Mohan, Int. Res. J. Pharmacy, 1, 201 (2010).

15. A. Seyedan, M.A. Alshawsh, M.A. Alshagga, S. Koosha and Z. Mohamed, Evid. Based Complement. Alternat. Med., 2015, 1 (2015); doi:10.1155/2015/973143.

16. G.E. Trease and W.C. Evans, Pharmacognosy, 13th ed., London: Bailliere Tindall, 1989.

17. J.B. Harborne, Phytochemical Methods, London: Chapman and Hall Ltd., 1973: 99-188.

18. A. Sofowara, Medicinal plants and traditional medicine in Africa, Ibadan, Nigeria: Spectrum Books Ltd, Ibadan, Nigeria, 1993: 289.

19. H. Chaudhary and D.R. Brocks, J. Pharm. Pharm. Sci., 16, 65 (2013); doi:10.18433/J37G7M.

20. S.Y. Hor, E. Farsi, M.F. Yam, N.M. Nuyah and M.Z. Asmawi, J. Med. Plants Res., 5, 2261 (2011).

21. Y. Chen, X. Zhang, B. Pan, X. Jin, H. Yao, B. Chen, Y. Zou, J. Ge and H. Chen, Lipids Health Dis., 9, 52 (2010); doi:10.1186/1476-511X-9-52.

22. P.W. Wilson, L.A. Zech, R.E. Gregg, E.J. Schaefer, J.M. Hoeg, D.L. Sprecher and H.B. Brewer Jr., Clin. Chim. Acta, 151, 285 (1985); doi:10.1016/0009-8981(85)90091-9.

23. T. Kalelioglu, A. Genc, N. Karamustafalioglu and M. Emul, Diabetes Metab. Syndr., 11(Suppl 1), S473 (2017); doi:10.1016/j.dsx.2017.03.038.

24. T.P. Johnston and W.K. Palmer, J. Cardiovasc. Pharmacol., 29, 580 (1997); doi:10.1097/00005344199705000-00003.

25. T.P. Johnston and W.K. Palmer, Biochem. Pharmacol., 46, 1037 (1993); doi:10.1016/00062952(93)90668-M.

26. C.R. Pal, V.N. Garge and V.J. Kadam, J. Medical Sci. Clinical Res., 3, 5000 (2015).

27. G.A. Mohamed, S.R.M. Ibrahim, E.S. Elkhayat and R.S. El Dine, Bull. Fac. Pharm. Cairo Univ., 52, 269 (2014); doi:10.1016/j.bfopcu.2014.05.001.

28. H.Y. Kim, D.M. Jeong, H.J. Jung, Y.J. Jung, T. Yokozawa and J.S. Choi, Biol. Pharm. Bull., 31, 73 (2008); doi:10.1248/bpb.31.73.

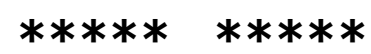

